\title{
Patterns of use of the Mental Health Act 1983, from 2007-2008 to 2016-2017, in two major London secondary mental healthcare providers
}

Sian Oram*, Craig Colling*, Megan Pritchard, Mizanur Khondoker, Daniela Fonseca de Freitas, Lucile Ter-Minassian, Johnny Downs, Brynmor Lloyd-Evans, Sarah Markham, Nomi Werbeloff, Chin-Kuo Chang, Sonia Johnson, Matthew Hotopf ${ }^{\dagger}$ and Richard D. Hayes ${ }^{\dagger}$

\section{Summary}

Trends in detention under the Mental Health Act 1983 in two major London secondary mental healthcare providers were explored using patient-level data in a historical cohort study between 2007-2008 and 2016-2017. An increase in the number of detention episodes initiated per fiscal year was observed at both sites. The rise was accompanied by an increase in the number of active patients; the proportion of active patients detained per year remained relatively stable. Findings suggest that the rise in the number of detentions reflects the rise of the number of people receiving secondary mental healthcare.

\section{Declaration of interest}

R.D.H. has received research funding from Roche, Pfizer, Janssen and Lundbeck. All other authors state no conflicts of interest.

\section{Keywords}

Compulsory admission; epidemiology; Mental Health Act.

\section{Copyright and usage}

(c) The Royal College of Psychiatrists 2019. This is an Open Access article, distributed under the terms of the Creative Commons Attribution-NonCommercial-NoDerivatives licence (http://creativecommons.org/licenses/by-nc-nd/4.0/), which permits noncommercial re-use, distribution, and reproduction in any medium, provided the original work is unaltered and is properly cited. The written permission of Cambridge University Press must be obtained for commercial re-use or in order to create a derivative work.
In the UK, detentions under the Mental Health Act 1983 (MHA) are reported to have risen steadily over the past three decades. ${ }^{1,2}$ This rise may be because of more people being detained, an increase in re-detention or both. However, it has not been possible, using national statistics, to measure directly any increase in the level of re-detention. This historical cohort study used patient-level data to explore trends in detention in two secondary mental health trusts in inner London from 2007-2008 to 2016-2017.

\section{Method}

Data were extracted from the Case Register Interactive Search (CRIS) systems at South London and Maudsley NHS Foundation Trust (SLAM) and Camden and Islington NHS Foundation Trust (C\&I). CRIS allows researchers to search and retrieve de-identified electronic mental health records within an approved governance framework. ${ }^{3,4}$ Individuals receiving active care by SLAM or C\&I at any time in a given fiscal year during the observation period (the 2007-2008 to 2016-2017 fiscal years at SLAM, inclusive, and the 2009-2010 to 2015-2016 fiscal years at C\&I) were included. Active care was defined as at least one face-to-face clinical encounter and/or a ward stay of at least 1 day. The population studied was working age adults (18-65 years, inclusive) from the SLAM and C\&I catchment areas (based on their residential or general practitioner's address). This excluded non-resident patients transferred out of area to receive care at SLAM and C\&I (these patients generally required national and specialist services and may not have been representative of locally resident patients), and patients detained to private hospitals. Homeless people are included. Part 2, part 3 and supplementary sections were included.

* S.O. and C.C. are joint first authors.

$\dagger$ M.H. and R.D.H. are joint senior authors.
Analyses focused on episodes of detention under the MHA ('detention episodes') and were conducted separately by site and fiscal year. Multiple sections of the MHA can be applied during a detention episode (for example a person may be detained for assessment under section 2 , then detained for treatment under section 3 ). If sections were used contiguously, they were a single episode. If there was a gap of more than 1 day between the end of one section and the start of a new section, this was a new episode.

Analyses considered whether any increase in total number of detention episodes was accounted for by more people being detained at least once, or by those who were detained experiencing this more frequently. We analysed (a) whether the number and proportion of patients detained at least once in a fiscal year increased year on year; and (b) whether, among those detained at least once in a fiscal year, the median number of detentions per person per year increased year on year. Analyses also considered duration of detention over time, in order to investigate whether any increase in detentions might be explained by a decrease in the number of days per detention.

Ethical approvals were provided by the Oxford C Research Ethics Committee (18/SC/0372 - SLAM) and the National Research Ethics Service Committee East of England - Cambridge Central (14/EE/0177 - C\&I). The SLAM and C\&I CRIS Oversight Committees approved the presented analyses.

\section{Results}

An increase in the number of detention episodes initiated per fiscal year was recorded at both Trusts, accompanied by an increase in the number of active patients. The proportion of active patients with at least one detention episode initiated during the fiscal year remained relatively stable (Table 1). At SLAM, the number of detention episodes increased from 1786 in 2007-2008 to 2374 in 2016-2017 
Table 1 Detention episodes at South London and Maudsley NHS Foundation Trust (SLAM) and Camden and Islington NHS Foundation Trust (C\&I)

\begin{tabular}{|c|c|c|c|c|c|c|}
\hline & \multicolumn{3}{|c|}{ SLAM NHS Foundation Trust } & \multicolumn{3}{|c|}{ C\&I NHS Foundation Trust } \\
\hline & $\begin{array}{l}\text { Detention } \\
\text { episodes, } n\end{array}$ & $\begin{array}{l}\text { Active } \\
\text { patients, } n\end{array}$ & $\begin{array}{c}\text { Active patients with } \\
\text { detention episode, } n(\%)\end{array}$ & $\begin{array}{l}\text { Detention } \\
\text { episodes, } n\end{array}$ & $\begin{array}{l}\text { Active } \\
\text { patients, } n\end{array}$ & $\begin{array}{c}\text { Active patients with } \\
\text { detention episode, } n(\%)\end{array}$ \\
\hline 2007-2008 & 1786 & 23226 & $1493(6.4)$ & - & - & - \\
\hline 2008-2009 & 1902 & 25219 & $1552(6.2)$ & - & - & - \\
\hline 2009-2010 & 2005 & 25673 & $1608(6.3)$ & 655 & 12131 & $562(4.6)$ \\
\hline 2010-2011 & 2097 & 26711 & $1665(6.2)$ & 718 & 12579 & $576(4.6)$ \\
\hline 2011-2012 & 2156 & 26063 & $1714(6.6)$ & 683 & 12483 & $553(4.4)$ \\
\hline 2012-2013 & 2213 & 26186 & $1768(6.8)$ & 673 & 13018 & $569(4.4)$ \\
\hline 2013-2014 & 2281 & 26922 & $1782(6.6)$ & 729 & 13490 & $606(4.5)$ \\
\hline 2014-2015 & 2350 & 27585 & $1875(6.8)$ & 639 & 13539 & $534(3.9)$ \\
\hline 2015-2016 & 2307 & 27706 & $1800(6.5)$ & 739 & 15123 & $629(4.2)$ \\
\hline 2016-2017 & 2374 & 27153 & $1857(6.8)$ & - & - & - \\
\hline
\end{tabular}

(a 33\% increase). During the same period the number of active patients increased from 23226 to 27153 (a 17\% increase) and the proportion detained changed from 6.4 to $6.8 \%$. The greatest increase occurred between 2007-2008 and 2009-2010, during which time there was a $12 \%$ increase in the number of detentions (from 1786 to 2005$)$ and an $11 \%$ increase in the number of active patients (from 23226 to 25 673). Data from C\&I were available for 20092010 to 2015-2016 only. There was more year on year variation, but an overall increase in the number of detention episodes from 655 to 739 (a 13\% increase). During the same period the number of active patients increased from 12131 to 15123 (a 25\% increase) and the proportion detained changed from 4.6 to $4.2 \%$.

The median number of detention episodes initiated per patient per fiscal year (our measure of re-detention) was stable over time at each Trust, the median being uniformly 1.0 in SLAM and in C\&I across the observation period. Different patterns were observed regarding the median duration of detention for active patients who had at least 1 day detained during the fiscal year. At SLAM the median duration of detention ranged from 39 to 54 days, with no clear trend over time. An increase in the median duration of detention was observed at C\&I, from 51 days in 2009-2010 to 61.5 days in 2015-2016. Further, when considering mean detention rates over the course of the study and between trusts, SLAM had a small increase (from 1.2 to 1.3 ) whereas these were stable at C\&I (at 1.2).

\section{Discussion}

Analyses showed a rising trend in detention under the MHA: the number of detention episodes increased by 33\% in SLAM between 2007-2008 and 2016-2017 and by 13\% in C\&I between 20092010 to 2015-2016. This increase is less pronounced than would have been expected from national data, which indicated that detentions increased by $47 \%$ nationally between 2005-2006 and 20152016..$^{5}$ This discrepancy may add weight to the hypothesis, advanced by the Care Quality Commission (CQC), that the increase in the rate of detention since 2010 is in part as a result of national data becoming more complete or to increased problems of double counting. ${ }^{6}$ This disparity may also reflect the increase in detention to private hospitals. ${ }^{5}$

Analyses also show that the increase in detentions at these Trusts may reflect an increase in the number of patients seen, with only modest changes to the proportion of patients being detained and little evidence that the same individuals were being detained more often over time. There are many potential reasons for such an increase, ${ }^{7,8}$ including - and as highlighted by the CQC - the increase in the local population. ${ }^{6}$ Population estimates indicate that the population served by C\&I increased by $14 \%$ between 2009 and 2015; the population served by SLAM similarly increased by $14 \%$ between 2007 and $2016 .^{9}$ Reasons may also include increased risk of developing a mental disorder because of social and economic change, increased help-seeking, ${ }^{7,8}$ and increased awareness and responsiveness to mental disorder among healthcare professionals. ${ }^{2}$ Further discussion of the findings is provided in Box 1 by a member of the NIHR Mental Health Policy Research Unit's Lived Experience Working Group.

\section{Box 1 NIHR Mental Health Policy Research Unit's lived experience working group commentary}

The findings of this study demonstrate that at both sites, both the number of detentions per year and the number of active patients rose over the considered time period. However, although the percentage of active patients detained under the Mental Health Act (MHA) increased slightly over the time period for one site, for the other it decreased slightly. As a patient who has experienced detention under the MHA, I am aware of the extent to which it can be both traumatising and damaging to one's confidence in mental health services. Detention is best avoided and is seldom experienced as therapeutic or helpful, even when perceived by an individual as having potentially saved their life. ${ }^{1}$ Therefore, it is positive that the overall number of detentions per individual did not increase over time; although it is unfortunate that at both sites the actual number of detentions per year did increase, and that at one site the mean length of detention per active patient detained also increased.

It is interesting to note also that at both sites the number of active patients increased and prompts the question as to why? In the discussion section the assumption is made that the number of active patients explains the increase in number of detentions per year, which is supported by the relative stability over the considered time period of the ratio of active patients detained. The discussion section also suggests possible causes for the increase in the number of active patients.

In one site the increase in number of detentions was matched by a proportionate increase in the population of the site, yet in the other the increase in the number of detentions was proportionately over twice the increase in the population of the site. It would be useful to determine the reasons behind this difference; for instance, whether it is because of differences in changes in mental health service provision and/or socio-environmental factors between the two sites over the considered time period.

This study suggests that the national rise in the rate of detentions under the MHA may be the result of increases in the number of people detained rather than increases in the number of detentions per individual. Given the adverse nature of patients' experiences of detention under the MHA, as evidenced by the current Independent Review of the $\mathrm{MHA}^{10}{ }^{10}$ this increase needs to be better understood with a view to reversing the current trend. Sarah Markham

(S. Markham, personal communication, 2019)

\section{Strengths and limitations}

CRIS offers several advantages over other types of routine data in investigating trends in MHA use. It provides patient-level, longitudinal data on demographic and clinical factors and on MHA 
detentions. In contrast with some national data that provides only the raw number of detentions, ${ }^{5}$ we could account for the number of individuals receiving care. However, there are limitations. Data are drawn from two London-based NHS trusts that are diverse both in terms of deprivation and ethnicity and may not be representative of wider national trends. ${ }^{5}$ Analyses do not account for potential confounders and it was beyond the scope of this study to investigate drivers of clinical decision-making or the impact of sociodemographic and economic factors.

\section{Implications}

In addition to highlighting the need for reliable routine data systems, this study reveals that the rising number of detentions may mainly reflect the rise of the number of people receiving mental healthcare. Future research should include investigation of this increase and multivariate analyses to investigate drivers of changes in the number of detentions and re-detentions over time and across different areas of England. Analyses should be replicated in trusts outside of London with access to systems such as CRIS and consideration given to developing similar systems in trusts lacking this type of resource.

Sian Oram (D), PhD, Senior Lecturer in Women's Mental Health, NIHR Mental Health Policy Research Unit \& Section for Women's Mental Health, Institute of Psychiatry, Psychology and Neuroscience, King's College London, UK; Craig Colling $\mathbb{D}$, Information Manager, NIHR Biomedical Research Centre, South London and Maudsley NHS Foundation Trust, UK; Megan Pritchard, CRIS Training and Development Lead, NIHR Biomedical Research Centre, South London and Maudsley NHS Foundation Trust, UK; Mizanur Khondoker, PhD, Senior Lecturer in Medical Statistics, Norwich Medical School, University of East Anglia, UK; Daniela Fonseca de Freitas, PhD, Postdoctoral Researcher, Department of Psychological Medicine, Institute of Psychiatry, Psychology and Neuroscience, King's College London, UK; Lucile Ter-Minassian, MSc, Research Worker, Department of Psychological Medicine Institute of Psychiatry, Psychology and Neuroscience, King's College London, UK; Johnny Downs, MRCPsych, PhD, Clinical Lecturer, Department of Child and Adolescent Psychiatry, Institute of Psychiatry, Psychology and Neuroscience, King's College London, UK; Brynmor Lloyd-Evans (D), PhD, Senior Lecturer, NIHR Mental Health Policy Research Unit, Division of Psychiatry, UCL, UK; Sarah Markham (D), PhD, Visiting Researcher, Department of Biostatistics, Institute of Psychiatry, Psychology and Neuroscience, King's College London, UK Nomi Werbeloff (iD, PhD, Senior Research Associate, Division of Psychiatry, UCL; and Camden and Islington NHS Foundation Trust, UK; Chin-Kuo Chang, PhD, Associate Professor, Department of Health and Welfare, University of Taipei, Taiwan; Sonia Johnson, DM, MRCPsych, Professor of Social and Community Psychiatry, NIHR Mental Health Policy Research Unit, Division of Psychiatry, UCL; and Camden and Islington NHS Foundation Trust, UK; Matthew Hotopf, PhD, FRCPsych, FMedSci, Professor of General Hospital Psychiatry, Department of Psychological Medicine, Institute of Psychiatry, Psychology and Neuroscience, King's College London, UK; Richard D. Hayes, PhD, Senior Lecturer, Department of Psychological Medicine, Institute of Psychiatry, Psychology and Neuroscience, King's College London, UK

Correspondence: Sian Oram. Email: sian.oram@kcl.ac.uk

First received 11 Jul 2019, final revision 15 Oct 2019, accepted 16 Oct 2019

\section{Funding}

This paper is based on independent research commissioned and funded by the National Institute for Health Research Policy Research Programme and the National Institute for Health Research (NIHR) Biomedical Research Centre at South London and Maudsley NHS Foundation Trust and King's College London. The views expressed are those of the author(s) and not necessarily those of the NHS, the NIHR, the Department of Health and Social Care or its arm's length bodies, and other government departments. CRIS is further supported by the Guy's and St Thomas' Trustees and the South London and Maudsley Trustees. R.D.H. receives salary support from the NIHR Mental Health Biomedical Research Centre at South London and Maudsley NHS Foundation Trust and King's College London. M.H. is an NIHR senior investigator.
N.W. is supported by the University College London Hospitals National Institute of Health Research Biomedical Research Centre.

\section{Data availability}

A dedicated CRIS Security Model has been developed to ensure that the ethical and legal rights of patients are protected. Access to CRIS data requires approval by the CRIS Oversight committee.

\section{Author contributions}

S.O. led the analysis and interpretation of data from Camden and Islington NHS Foundation Trust and drafted the first version of the manuscript. C.C. led data extraction, analysis and inter pretation for the South London and Maudsley NHS Foundation Trust site. M.P. made substantial contributions to data extraction and the interpretation of the data structure and results. M.K. provided guidance on statistical aspects of the paper and assisted with interpretation of the analysis. D.F.d.F. made substantial contributions to the interpretation of data. L.T.-M. made substantial contributions to the interpretation of data. J.D. contributed to the interpretation of data and provided guidance on the underlying clinical practice reflected in the data. B.L.-E. made substant Islington NHS Foundation Trust and to the interpretation of findings. S.M. made substantial contributions to the interpretation of data. N.W. led on data acquisition for analysis conducted at Camden and Islington NHS Foundation Trust. C.-K.C. provided epidemiological support and assisted with study design. S.J. made substantial contributions to the design of analysis conducted at Camden and Islington NHS Foundation Trust and to the interpretation of findings M.H. made substantial contributions to the overall conceptualisation and design of the work to the interp retation of data R. H. Ied the conceptrisation $\mathrm{N}$ and to the interpretation of data from South London and Maudsley NHS Foundation Trust. All
authors drafted and/or critically revised the manuscript and approved the final version of the manuscript.

\section{References}

1 Hotopf M, Wall S, Buchanan A, Wessely S, Churchill R. Changing patterns in the use of the Mental Health Act 1983 in England, 1984-1996. Br J Psychiatry 2000; 176: 479-84

2 Keown $\mathrm{P}$, Murphy $H$, McKenna D, McKinnon I. Changes in the use of the Mental Health Act 1983 in England 1984/85 to 2015/16. Br J Psychiatry 2018; 213: 595-9.

3 Stewart R, Soremekun M, Perera G, Broadbent M, Callard F, Denis M, et al. The South London and Maudsley NHS Foundation Trust Biomedical Research Centre (SLAM BRC) case register: development and descriptive data. BMC Psychiatry 2009; 9: 51.

4 Perera G, Broadbent M, Callard F, et al. Cohort profile of the South London and Maudsley NHS Foundation Trust Biomedical Research Centre (SLaM BRC) Case Register: current status and recent enhancement of an electronic mental health record-derived data resource. BMJ Open 2016; 6: e008721.

5 Community and Mental Health Team, NHS Digital. Inpatients Formally Detained in Hospitals Under the Mental Health Act 1983, and Patients Subject to Supervised Community Treatment: 2015/2016, Annual Figures. NHS Digital, 2016.

6 Care Quality Commission. Monitoring the Mental Health Act in 2016/17. Care Quality Commission, 2018

7 McManus S, Bebbington P, Jenkins R, Brugha T (eds.). Mental Health and Wellbeing in England: Adult Psychiatric Morbidity Survey 2014. NHS Digital, 2016.

8 Baker C. Mental Health Statistics for England: Prevalence, Services and Funding. House of Commons, 2018.

9 Office for National Statistics. Estimates of the population for the UK, England and Wales, Scotland and Northern Ireland. ONS, 2019 (https://www.ons gov. uk/peoplepopulationandcommunity/populationandmigration/populationesti mates/datasets/populationestimatesforukenglandandwalesscotlandandnorth ernireland).

10 Department of Health and Social Care. Independent Review of the Mental Health Act: Interim Report. Department of Health and Social Care, 2018.

OPEN 